\title{
Treatment of a mouse model of collagen antibody-induced arthritis with human adipose-derived secretions
}

\author{
Sinead P. Blaber ${ }^{1,2}$, Rebecca A. Webster ${ }^{1,2}$, Edmond J. Breen ${ }^{3}$, Graham Vesey $^{2}$, \\ Benjamin R. Herbert ${ }^{1,2 *}$ \\ ${ }^{1}$ Department of Chemistry and Biomolecular Sciences, Macquarie University, North Ryde, Australia; \\ ${ }^{*}$ Corresponding Author: benjamin.herbert@mq.edu.au \\ ${ }^{2}$ Regeneus Ltd., Gordon, Australia \\ ${ }^{3}$ Australian Proteome Analysis Facility, Macquarie University, North Ryde, Australia
}

Received 2 August 2013; revised 2 September 2013; accepted 9 September 2013

Copyright (C) 2013 Sinead P. Blaber et al. This is an open access article distributed under the Creative Commons Attribution License, which permits unrestricted use, distribution, and reproduction in any medium, provided the original work is properly cited.

\section{ABSTRACT}

The use of adipose-derived cells as a treatment for a variety of diseases is becoming increasingly common. These therapies include the use of cultured mesenchymal stem cells (MSCs) and freshly isolated stromal vascular fraction (SVF) alone, or in conjunction with other cells such as adipocytes. There is a substantial amount of literature published on the therapeutic properties of MSCs and their secretions as the main driver of their therapeutic effect. However, there is little data available on the therapeutic potential of secretions from SVF, either with or without adipocytes. We investigated the ability of secretions from human adipose SVF alone and the SVF co-cultured with adipocytes as a proxy for cell therapy, to ameliorate an inflammatory disorder. This ethics approved study involved the treatment of collagen antibody-induced arthritis (CAIA) in mice with secretions from SVF, SVF co-cultured with adipocytes, or a vehicle control via both intravenous (IV) and intramuscular (IM) routes. Treatment outcome was assessed by paw volume, ankle size and clinical arthritis score measurements. Serum samples were obtained following euthanasia and analysed for a panel of 32 mouse cytokines and growth factors. The dose and timing regime used for the IM administration of both human secretion mixtures did not significantly ameliorate arthritis in this model. The IV administration of SVF adipocyte co-culture secretions reduced the paw volume, and significantly reduced the ankle size and clinical arthritis score when compared to the IV vehicle control mice. This was a superior therapeutic effect than treatment with SVF secretions. Furthermore, treatment with SVF adipocyte coculture secretions resulted in a significant reduction in serum levels of key cytokines, IL-2 and VEGF, involved in the pathogenesis of rheumatoid arthritis. Therefore, the SVF cocultured with adipocytes is an attractive therapeutic for inflammatory conditions.

Keywords: Collagen Antibody-Induced Arthritis (CAIA); Stromal Vascular Fraction (SVF); Adipocytes; Co-Culture; Secretions; Cytokines; Growth Factors; Bio-Plex; Rheumatoid Arthritis

\section{INTRODUCTION}

The use of adipose-derived cells, including cultured adipose-derived mesenchymal stem cells (MSCs), uncultured stromal vascular fraction (SVF) and SVF combined with other cells such as adipocytes, is becoming increasingly common for the treatment of a variety of diseases. There are currently two distinct pathways, defined by the regulatory environment, by which cell therapies can proceed down to clinical use in a number of major jurisdictions, including Australia, the UK and some European countries. The first involves significant safety and efficacy trials and is usually required for cell therapies that involve allogeneic cells and in vitro expansion or other forms of manipulation before administering the cells to the patient. The second is a medical exemption regulatory category whereby a registered medical practitioner can treat a patient in a clinic or hospital set- 
ting with a custom-made therapeutic for that patient providing the entire procedure is under the supervision of the medical practitioner [1]. This alternative pathway encompasses the use of autologous, freshly harvested tissue that has only undergone minimal manipulation to produce a cell population for therapeutic use in an inclinic procedure. The use of freshly isolated adiposederived cell populations such as the SVF, alone or in combination with adipocytes, falls within this medical exemption category. Consequently, the use of such cell populations as autologous therapeutics is increasing.

Historically, the focus of cellular therapy has been defining the ability of stem cells, including MSCs, to differentiate into cells of the target issue type and their subsequent potential therapeutic use. In recent years there has been a shift in focus towards using fresh uncultured cells for therapeutic use such as the SVF with or without adipocytes partly due to the aforementioned regulatory reasons. Furthermore, the realization that secretions are a major driver of the therapeutic effect in mesenchymal cellular therapy [2-4] has led to an increased focus on the secretion capabilities of cells. Consequently the secretion profiles produced by these isolated and mixed cell populations are of interest in order to delineate functional differences and guide potential therapeutic use of cellular therapies. These adipose-derived secretions may also be of importance for future off-the-shelf secretion based therapeutics for use when cellular therapy may not be possible or appropriate, or in some cases may be used in conjunction with cellular therapy.

Adipose tissue is comprised of a variety of different cell types, which reside in close proximity to each other in vivo. This allows for substantial cross-talk between these different cell types though cell-cell contact, surface molecular receptors and secreted factors. This communication between the cells is essential for the normal functioning of adipose tissue and this complex-reciprocal signaling has been demonstrated by in vitro co-culture experiments [5-7]. We have previously demonstrated that unique secretion profiles can be obtained from different adipose-derived cell populations [8]. In particular, we demonstrated that the co-culture of adipocytes with the SVF results in the cells acting in a synergistic manner and consequently increasing the production of 9 cytokines when compared to culturing the SVF alone [8]. For example, IL-1 R $a$ was produced in significantly increased levels by the co-culture of SVF with adipocytes versus the SVF alone. IL-1R $a$ is one of the most important naturally occurring anti-inflammatory cytokines because it blocks the actions of the potent pro-inflammatory cytokine, IL- $1 \beta$. Consequently, IL-1R $a$ is currently being trialed as a treatment for diseases with a significant inflammatory component, such as autoimmune disorders.

The administration of a single cytokine or growth fac- tor has been trialed in animals with induced diseases and humans with clinical conditions. This treatment approach has frequently resulted in the development of adverse reactions [9-11]. For example, the administration of IL12 to cancer patients in a Phase II clinical trial caused serious side effects including renal, neurological, hepatic hematopoietic and cardiac toxicities resulting in the hospitalization of 12 patients and the death of 2 patients [9]. In this case, the administration of IL-12 caused an inappropriate immune response and a profound up-regulation of IFN- $\gamma$ [9]. In contrast, some biologics, in particular the administration of recombinant IL-1R $a$, have not demonstrated any efficacy, or only a modest effect, for autoimmune disorders [see 12 and references therein]. To this end, studies have demonstrated that treatment with two bioactive factors results in a superior therapeutic outcome than the administration of these factors individually $[13,14]$. There are numerous in vitro studies illustrating the immuno-modulatory, angiogenic, mitogenic, anti-apoptotic, and anti-scarring properties of both adipose-derived and bone marrow-derived MSCs [see 15 and references therein]. Furthermore, administration of the secretions from these MSCs has been shown to improve corneal wound healing following chemical burns [16], and recovery from myocardial infarctions [17] and ischemic brain injuries [18]. These studies demonstrate the therapeutic potential of MSC secretions for the treatment of non-autoimmune conditions, however there is little data available on the ability of secretions to ameliorate autoimmune conditions. Additionally, there is limited data available on the treatment of conditions in vivo with cells or secretions from mixed cell populations such as the SVF, alone or in combination with adipocytes. As we have previously demonstrated that the in vitro co-culturing of adipocytes with the SVF results in a distinct secretion profile when compared to culturing the SVF alone [8], the in vivo comparison of this result is of interest. Given the promising data available on conditioned media for injury models, we were interested in its efficacy for autoimmune conditions. Our interest was to understand the efficacy of human adipose derived cells for inflammatory autoimmune conditions. Cultured MSCs do not usually elicit an immune response, even when used as a xeno-transplant. This would not be the case, however, for a mixed population of human cells in immuno-competent mice. In order to test the likely therapeutic effect of human SVF with and without adipocytes, we chose to use conditioned media alone, thus avoiding any immunological issues with human cells.

Collagen antibody-induced arthritis (CAIA) in mice is an inflammatory disorder that shares many pathological and histological similarities with clinical rheumatoid arthritis in humans, including synovitis with infiltration of polymorphonuclear and mononuclear cells, pannus 
formation, fibrosis cartilage degradation and bone erosion [19]. Furthermore, significant swelling and redness is observed in the paws of mice suffering from CAIA [20]. CAIA is induced by the administration of a cocktail of 5 anti-type II collagen antibodies, followed by a lipopolysaccharide (LPS) injection three days later. The subsequent administration of LPS following the antibody cocktail not only increases the severity of the arthritis through the induction of pro-inflammatory cytokines and complement component activation, but also reduces the amount of monoclonal antibody required to induce the arthritis in this model [20 and references therein, 21]. Arthritis in the CAIA model development is rapid and relatively synchronized through the synchronization of antibody administration [19].

The primary objective of this work was to perform an in vivo study to investigate the ability of human adipose-derived secretions, as a proxy for human SVF cell therapy, to ameliorate an inflammatory disorder. The SVF cell secretions, with or without adipocyte co-culture, are a complex mixture of proteins and other signaling factors. Many of the cytokines secreted by adipose SVF have pleiotropic effects, which combined with the large number of cytokines would make any attempt at elucidating the mechanism of action very difficult. The goal of this study was to investigate the likely therapeutic benefit of SVF cellular therapy rather than an attempt to identify key cytokines. As there is limited in vivo data available on the use of secretions from mixed cell populations such as adipose SVF, we chose to pursue a preliminary study to compare the ability of human SVF co-cultured with adipocytes (referred to as SVF adipocyte co-culture) and SVF secretions to ameliorate arthritis in a CAIA model in mice. Furthermore, we investigated whether administration via the intravenous (IV) or intramuscular (IM) route had any effect on the ability of these adipose-derived secretions to reduce the severity of arthritis in this mouse model.

\section{MATERIALS AND METHODS}

\subsection{Production of Secretions from Human Lipoaspirate}

This research, involving obtaining human adipose tissue, and administering the resultant human secretions to animals, was approved by the Macquarie University human research ethics committee. Written consent was obtained from the patient who provided their lipoaspirate sample after undergoing a routine liposuction procedure for cosmetic reasons. The lipoaspirate sample was processed as previously described [8]. Briefly, $200 \mathrm{~g}$ of lipoaspirate was digested with $0.5 \mathrm{mg} / \mathrm{mL}$ collagenase (Lomb Scientific, USA) in saline in a $37^{\circ} \mathrm{C}$ water bath for $30 \mathrm{~min}$ with periodic mixing. The digested samples were passed through an $800 \mu \mathrm{m}$ mesh and centrifuged at $1500 \times \mathrm{g}$ for $5 \mathrm{~min}$ to obtain the pelleted cells (SVF) and floating adipocytes. The floating free lipid layer was aspirated and discarded. The adipocyte and SVF fractions were washed separately with saline and centrifuged at $1500 \times \mathrm{g}$ for $5 \mathrm{~min}$. The SVF pellet was resuspended in our Standard Media that consisted of high glucose Dulbecco's Modified Eagle Medium (HG DMEM; Invitrogen, USA) supplemented with $10 \%$ foetal bovine serum (FBS; Bovogen, Australia) and 1\% PenicillinStreptomycin solution (Invitrogen, USA). A portion of the SVF pellet was filtered through a $35 \mu \mathrm{m}$ nylon mesh topped tube (Becton Dickinson, USA). SVF samples were enumerated and the viability determined in TruCount tubes (Becton Dickinson, USA) containing isoflow (Becman Coulter, USA) propidium iodide (10 $\mu \mathrm{g} / \mathrm{mL}$; Sigma, USA) and Syto11 (1 $\mu \mathrm{M}$; Invitrogen, USA) using a FacsScan flow cytometer (Becton Dickinson, USA). The total number of viable cells in the SVF pellet was determined.

Two $\mathrm{T} 175 \mathrm{~cm}^{2}$ culture flasks were each seeded with 29 million viable SVF cells. The SVF adipocyte co-culture flask also received $30 \mathrm{~mL}$ of adipocytes. As the adipocytes transferred to the SVF adipocyte co-culture flask were floating saline, the SVF flask received $30 \mathrm{~mL}$ of saline. The volume in each $T 175 \mathrm{~cm}^{2}$ flask was normalized to a total of $80 \mathrm{~mL}$ with Standard Media. The flasks were incubated at $37^{\circ} \mathrm{C}$ with $5 \% \mathrm{CO}_{2}$ for 72 hours and the conditioned medium collected, centrifuged at $4980 \mathrm{x} g$ for $10 \mathrm{~min}$ and stored at $-80^{\circ} \mathrm{C}$. These conditioned medium samples were thawed, filter sterilized using $0.22 \mu \mathrm{m}$ syringe filters, aliquoted and frozen at $-80^{\circ} \mathrm{C}$. These aliquoted samples were administered to mice suffering from CAIA.

\subsection{Collagen Antibody-Induced Arthritis- Mouse Model}

A commercial company, TetraQ, based at the University of Queensland, conducted this CAIA trial. The University of Queensland's animal ethics committee approved this trial involving the administration of human adipose-derived secretions to mice suffering from CAIA. The trial was blinded and was performed at the TetraQ premises in accordance with the guidelines set out in the Australian Code of Practice for the Care and Use of Animals for Scientific Purposes, 7th edition, 2004. Nulliparous and non-pregnant female Balb/c (BALB/ cJAsmu) mice at $6-8$ weeks of age were used in this trial. The mice had access to rat and mouse pellets (Specialty feeds, Australia), chewing sticks (Able Scientific, Australia), Alphatwists (Tecniplast, Australia) and water ad libitum throughout the study. The housing temperature throughout the study was $23^{\circ} \mathrm{C} \pm 3^{\circ} \mathrm{C}$, with a humidity of $30 \%-70 \%$, a 12 -hour light/dark cycle and a 
minimum of 15 air changes per hour. The mice were allowed to acclimatize to these housing conditions for 3 days prior to commencement of the trial.

At day zero, each mouse (total of 36 mice) received an intravenous injection of $1.5 \mathrm{mg}(150 \mu \mathrm{L})$ of an anti-type II collagen 5 clone antibody cocktail (Chondrex Inc., Australia). On day 3, mice received an intraperitoneal injection of $80 \mu \mathrm{L}(40 \mu \mathrm{g} /$ mouse $)$ of LPS. The trial design consisted of 6 groups, each containing 6 mice (Table 1). Groups 1, 2, and 3 received $100 \mu \mathrm{L}$ of human SVF adipocyte co-culture secretions, human SVF secretions or the vehicle control (containing HG DMEM and $1 \% \mathrm{P} / \mathrm{S}$ ) respectively via the intravenous (IV) route on days 6,7 and 8 . Groups 4,5 and 6 received $50 \mu \mathrm{L}$ of human SVF adipocyte co-culture secretions, human SVF secretions or vehicle control respectively via the intramuscular (IM) route every second day for 4 days (days 6 , 8,10 and 12).

The mice were monitored throughout the trial period ( 2 weeks) and paw volume, ankle size and clinical arthritis score measurements were taken on days 0 and 2 - 13 . The measurements were taken prior to administration of the collagen antibody cocktail (days 0) and LPS (day 3) and prior to administration of vehicle and the test articles (human SVF adipocyte co-culture secretions and SVF secretions). The paw volume of each mouse was measured using a plethysmometer. Plethysmometers are routinely used to measure small volume changes in rodent paws by measuring the water displacement following immersion of the paw in a specialized chamber which is attached to a digital monitor [22]. The paw size was measured using microcalipers across the hillock (ankle joint) of each hindpaw. The mice were assessed for the severity of arthritis using a standard scale [23] (0-normal; 1 -mild redness, slight swelling of ankle or wrist, redness and swelling limited to individual joints; 2 moderate swelling of ankle or wrist, redness in more than one joint, 3-severe swelling including some digits, ankle or foot; 4-maximal swelling and inflamed, involveing multiple joints). Following euthanasia with Lethabarb at day 14, blood was collected from all mice via- cardiac puncture. Blood was allowed to clot at room temperature for $30 \mathrm{~min}$ prior to centrifuging at $2500 \times \mathrm{g}$ for $10 \mathrm{~min}$ to obtain serum. Serum samples from agematched naïve mice were also obtained as control samples. Serum samples were stored at $-80^{\circ} \mathrm{C}$ prior to analysis using Bio-Plex technology.

\subsection{Bio-Plex Analysis of Serum Samples}

A total of 32 mouse cytokines were measured in the serum samples of mice treated IV with the vehicle control or human secretion mixtures to investigate the effect of the various treatment regimes on the mice. The SVF and SVF adipocyte co-culture secretion mixtures, vehicle control, media control and all IV treated mouse serum samples (including the naïve mouse serum samples) were filtered through $0.20 \mu \mathrm{m}$ Nanosep MF Centrifugal Devices with Bio-Inert ${ }^{\circledR}$ Membrane (Pall Scientific, USA) for $5 \mathrm{~min}$ at $9000 \mathrm{x}$ g. Samples $(50 \mu \mathrm{L})$ of each filtered sample were analysed using the Bio-Plex Pro Mouse Cytokine 23-plex and Bio-Plex Pro Mouse Cytokine 9-plex assays (Bio-Rad, USA) according to the manufacturers instructions. The washing steps were performed on the Bio-Plex Pro II magnetic wash station and the data was acquired using the Bio-Plex 200 system with version 5.0 software (Bio-Rad, USA). Mouse IL-1R $a$ cross-reacts with the human IL-1R $a$ antibody in the BioPlex Pro Human Cytokine 27-plex assay. Therefore IL$1 \mathrm{Ra}$ was measured in the mouse serum samples using this human kit and the same methodology described above.

\subsection{Data Analysis}

\subsubsection{Data Analysis of CAIA Primary Outcome Measurements}

The primary outcome measures, paw volume $\left(\mathrm{cm}^{3}\right)$, ankle size ( $\mathrm{mm})$, and clinical arthritis score in each group are presented as the mean \pm standard error of the mean $(\mathrm{SEM})$. The Delta $(\Delta)$ clinical arthritis score, paw volume and paw size was calculated by subtracting the pretreatment score from the post-treatment scores. Negative

Table 1. Trial design of treatment groups of mice suffering from collagen antibody-induced arthritis.

\begin{tabular}{|c|c|c|c|c|}
\hline Group (6 mice/group) & Treatment & Route of administration & Volume administered & Treatment regime \\
\hline Group 1 & SVF adipocyte co-culture secretions & & & \\
\hline Group 2 & SVF secretions & Intravenous (IV) & $100 \mu \mathrm{L}$ & Days 6,7 and 8 \\
\hline Group 3 & Vehicle & & & \\
\hline Group 5 & SVF secretions & Intramuscular (IM) & $50 \mu \mathrm{L}$ & Days $6,8,10$ and 12 \\
\hline Group 6 & Vehicle & & & \\
\hline
\end{tabular}


values were given a value of 0 . The Area Under the $\Delta$ clinical arthritis score, paw volume and paw size versus day curves were determined for each group as a measure of the extent of action and duration of arthritis. The non-parametric Dunnett's Multiple Comparison test was performed on $\Delta$ clinical arthritis score, paw volumeand paw size area under the curve (AUC) values for groups of mice administered either SVF adipocyte coculture secretions or SVF secretions relative to the corresponding values for animals administered vehicle controls. The Graphpad Prism data analysis software package (version 5.03) was used for all data and statistical analysis. The statistical significance criterion was $\mathrm{P}<0.05$. A Welch two-tailed t-test was performed on the clinical score raw data between mice in the IV SVF adipocyte co-culture and SVF secretion treatment groups.

\subsubsection{Data Analysis of Bio-Plex Datasets}

The human SVF adipocyte co-culture and SVF secretion mixtures were run on both mouse Bio-Plex kits as controls. We observed some cross-reactivity, however, this should have no impact on the study because the circulating half-life of cytokines is usually minutes to hours, although it differs for individual cytokines [24]. In this study, the serum was collected from mice 6 days after the last IV administration of human secretions. Therefore, we have assumed $0 \%$ of the human cytokines administered to the mice remained in their circulation at the point of euthanasia.

The mean \pm SEM values of all cytokines measured were determined for each of the groups $(n=6$ for all IV treatment groups, $\mathrm{n}=3$ for naïve mice). A two-tailed t-test was used to test the significance between the average concentrations of each cytokine in the IV treatment groups (SVF adipocyte co-culture secretions and SVF secretions) to the vehicle control group. p-values $<0.05$, denoted with $\mathrm{a}^{*}$, were considered statistically significant compared to the corresponding vehicle control group. Additionally, a two-tailed t-test was also used to test the significance between the average concentrations for each cytokine in the treatment groups (SVF adipocyte coculture secretions and SVF secretions) and the vehicle control group, when compared to the naïve mice serum. These statistically significant results with a p-value $<$ 0.05 are denoted with $\hat{a}$.

\section{RESULTS}

\subsection{Primary Outcome measures}

\subsubsection{IM}

Figure 1 illustrates that administration of SVF adipocyte co-culture and SVF secretions IM slightly reduced the primary outcome measures of paw volume (Figure 1(a)), ankle size (Figure 1(d)) and clinical arthritis score (Figure 1(g)) when compared to the mice that received the vehicle control IM. However, an analysis of the $\Delta$ area under the curve ( $\triangle \mathrm{AUC})$ results for these primary outcome measures (Figures 1(c), (f) and (i) over the course of the trial demonstrates that the reduction in these measurements following treatment with SVF adipocyte co-culture or SVF secretions was not statistically sig- nificant. Whilst these results were promising, a better clinical outcome may be obtained after optimization of the dose and timing of the IM treatment regime with SVF adipocyte co-culture or SVF secretions.

\subsubsection{IV}

Figure 1 contains the results of the primary outcome measures from the mice treated with the vehicle control, SVF adipocyte co-culture secretions or SVF secretions via the IV route. From these graphs it is evident that administration of SVF adipocyte co-culture and SVF secretions reduced the paw volume (Figure 1(b)), ankle size (Figure 1(e)) and clinical arthritis scores (Figure 1(f)) of mice suffering from CAIA when compared to the vehicle control group. In particular, the average clinical arthritis score of the mice treated with SVF adipocyte co-culture secretions was the only treatment group to achieve a reduction in clinical arthritis score to baseline following the establishment of severe arthritis (Figure 1(h)). The analysis of the $\triangle \mathrm{AUC}$ results demonstrated that the average paw volume throughout the course of the study was reduced in the mice treated IV with SVF adipocyte co-culture secretions when compared to the IV vehicle control although this reduction was not statistically significant (Figure 1(c)). The $\triangle \mathrm{AUC}$ ankle size was significantly reduced in both the SVF adipocyte co-culture and SVF secretion treatment groups when compared to the IV vehicle control (Figure 1(f)). Looking at an overall measure of effect, the IV administration of the SVF adipocyte co-culture secretions resulted in a significant reduction in the $\triangle \mathrm{AUC}$ clinical arthritis score (Figure 1(i)). Furthermore, the SVF adipocyte co-culture secretions significantly ( $p$-value $=0.005$ using a Welch twosided t-test) reduced the clinical arthritis scores of mice when compared to the SVF secretion treatment group.

These results demonstrate that the IV administration of secretions derived from adipose cell populations resulted in the reduction of all measurements of arthritis in CAIA mice when compared to the vehicle control mice. Specifically, IV treatment with secretions from the SVF co-cultured with adipocytes produced the largest effect, with a reduction in the paw volume and a significant reduction in the ankle size and clinical arthritis score when compared to the vehicle control group.

\subsection{Serum Levels of Mouse Cytokines and Growth Factors}

For these experiments the serum from naïve mice was 
used as an indicator of normal physiological cytokine levels. Figure 2 contains the serum levels of the pro- and anti-inflammatory cytokines measured in the naïve mice, and the mice treated IV with the vehicle control, SVF adipocyte co-culture or SVF secretions. IL- $1 \alpha$ was present in significantly higher levels in the serum of the mice treated with SVF adipocyte co-culture secretions when compared to the IV vehicle control group (Figure 2(a)). Additionally the levels of IL-1 $\alpha$ in the mice treated with SVF adipocyte co-culture and SVF secretions were significantly higher than the levels measured in the naïve mice (Figure 2(a)). The concentration of IL-1 $\beta$ measured in the vehicle control and both secretion treatment groups were significantly higher than the physiological levels seen in the naïve mice (Figure 2(b)). The level of IL-5 measured in the vehicle control group was significantly higher than the level measured in the naïve mice (Figure 2(c)). In comparison to the vehicle control mice, the serum of mice treated with SVF adipocyte co-culture and SVF secretions contained significantly reduced levels of IL-5 (Figure 2(c)). TNF- $\alpha$ was measured in significantly higher levels in the vehicle control and SVF adipocyte co-culture groups when compared to the naïve mice (Figure 2(d)). Whilst treatment with SVF and SVF adipocyte co-culture secretions increased the levels of the anti-inflammatory cytokine, IL-1R $a$, this increase was not statistically significant (Figure 2(e)). The levels of IL-10 and IL-13 were significantly increased in the mice treated with SVF adipocyte co-culture secretions when compared naïve mice (Figures 2(f) and (g) respectively). IL-2 and IL-6 are considered to have pro-inflammatory or anti-inflammatory roles under different circumstances. Both IL-2 and IL-6 were present in significantly increased concentrations in the serum of the vehicle control group mice when compared to the naïve mice (Figures 2(h) and (i) respectively). However, sig-
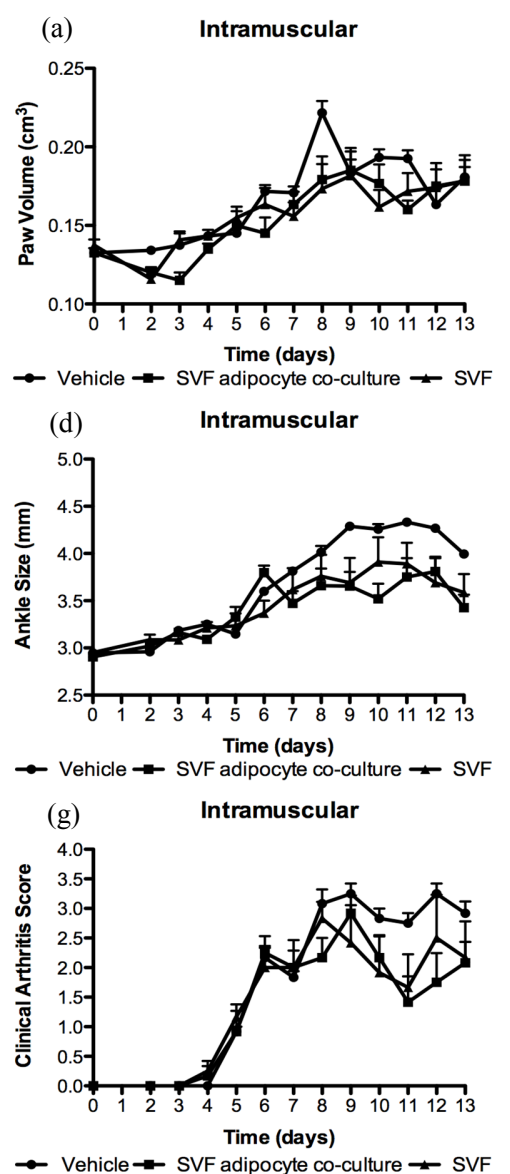

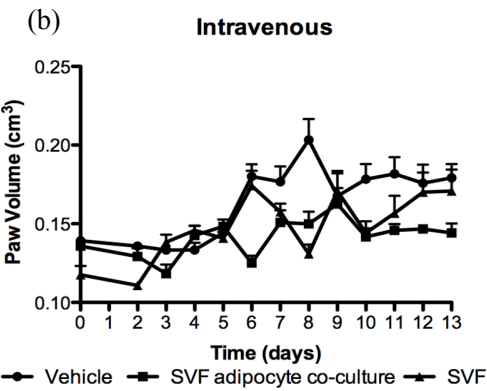

(e) Intravenous
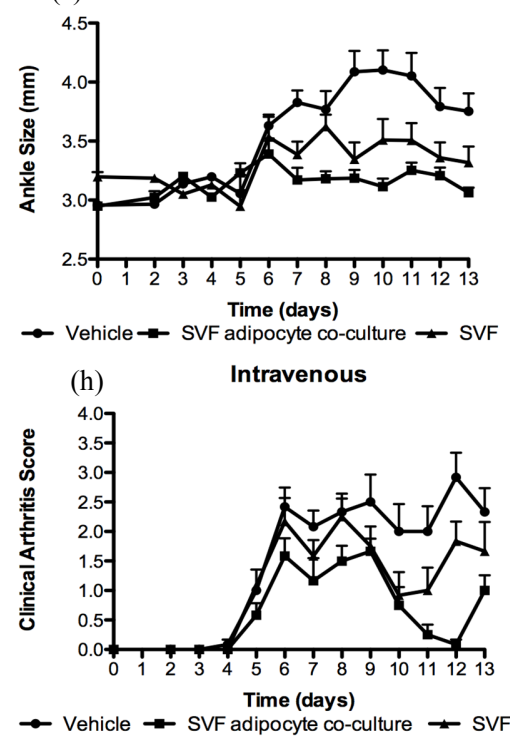
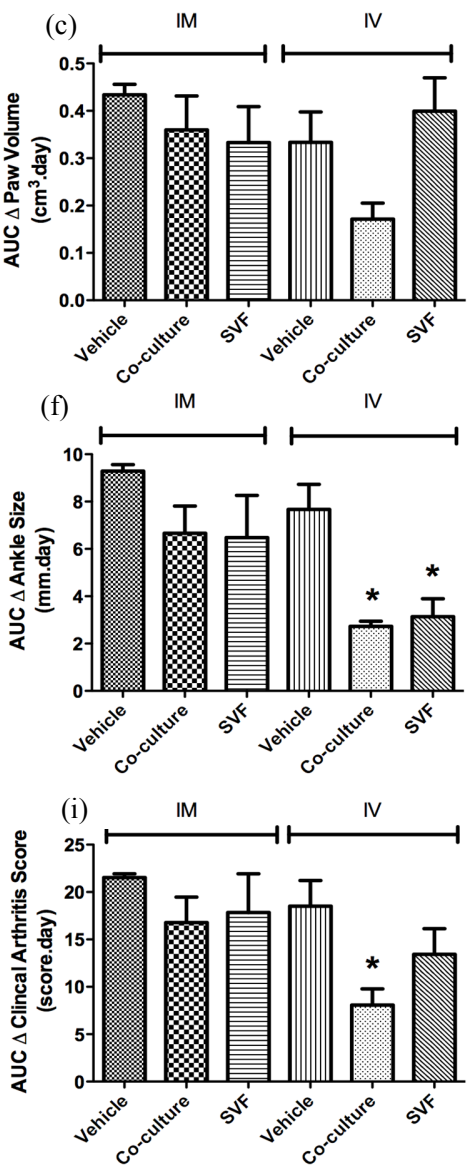

Figure 1. Primary outcome measures of mice suffering from collagen antibody-induced arthritis treated with secretions from SVF co-cultured with adipocytes, SVF secretions or vehicle controls. Mice were induced with collagen antibody-induced arthritis and subsequently treated with a vehicle control, SVF adipocyte co-culture secretions or SVF secretions via the intramuscular (IM) or intravenous (IV) route of administration. Paw volume (a)-(b), ankle size (d)-(e) and clinical arthritis score (g)-(h) were measured in the mice throughout the course of the trial. These results are expressed as the absolute mean \pm standard error of the mean (SEM; $n=$ 6). The mean \pm SEM $\Delta$ area under the curve $(\triangle A U C)$ trial results for the paw volume (c), ankle size (f), and clinical arthritis score (i) were calculated for each treatment group. The $\triangle \mathrm{AUC}$ results obtained from each treatment group were compared to the corresponding vehicle control administered by the same route. $a^{*}$ denotes a p-value of $<0.05$ when compared to the IV vehicle control group. 
(a)

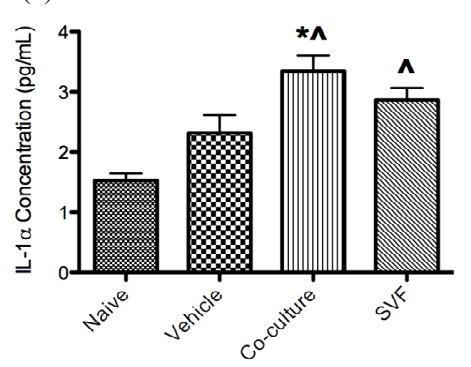

(d)

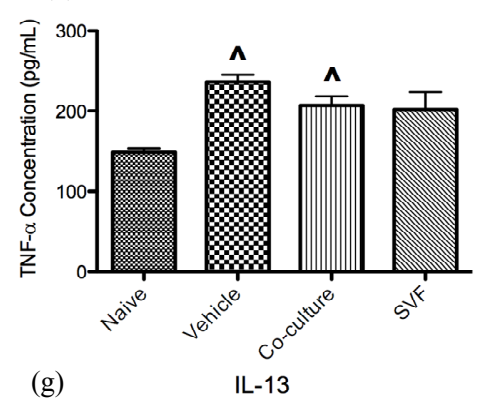

(g)

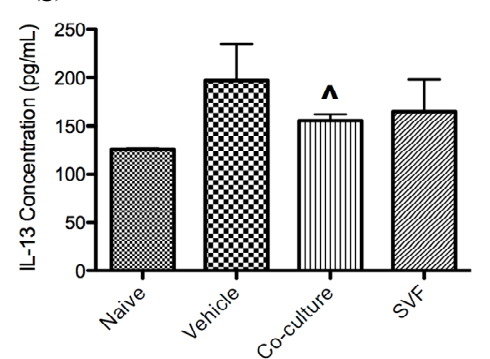

(b)

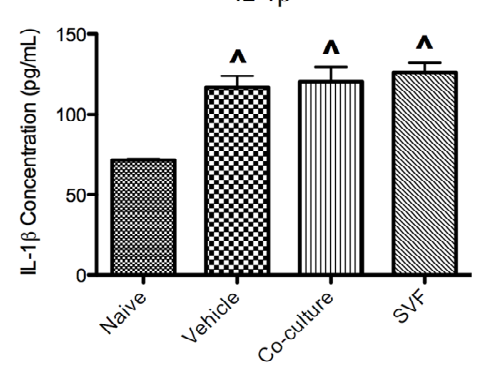

(e)
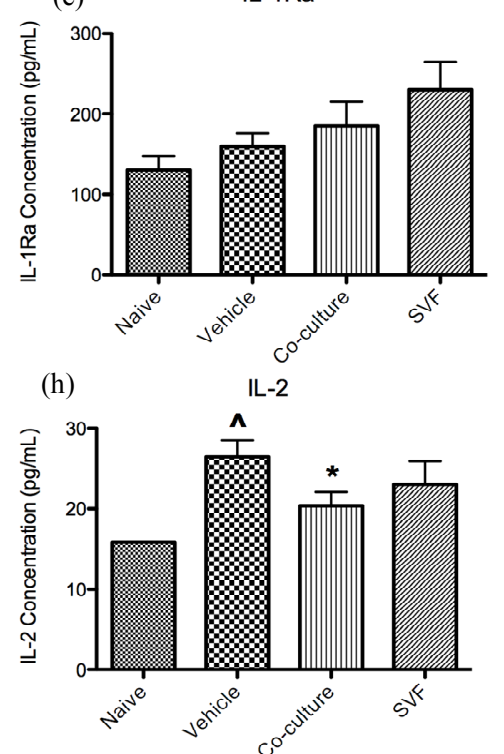

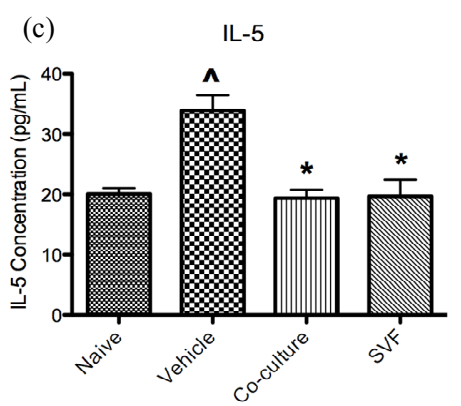

(f)

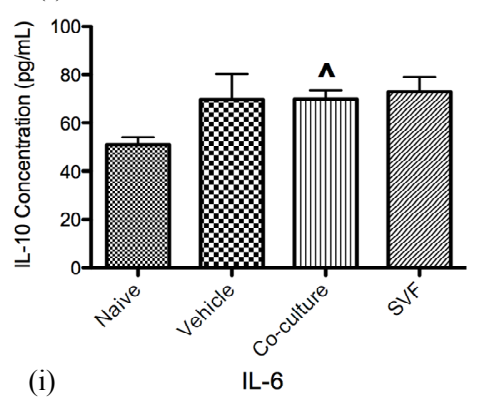

(i)

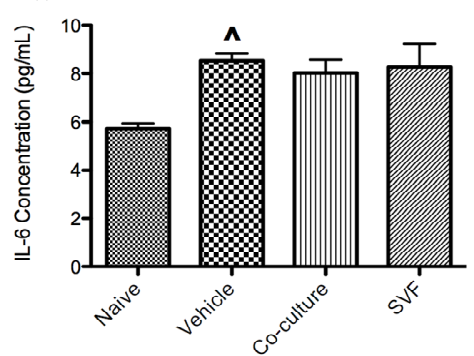

Figure 2. Serum levels of pro- and anti-inflammatory cytokines measured in mice suffering from collagen antibody-induced arthritis following IV treatment with secretions from the SVF co-cultured with adipocytes, SVF secretions or the vehicle control. Serum samples were collected following euthanasia of the mice in all IV treatment groups at the completion of the trial and from naïve mice. The results of the pro-inflammatory cytokines, IL-1 $\alpha$ (a), IL-1 $\beta$ (b), IL-5 (c) and TNF- $\alpha$ (d), the anti-inflammatory cytokines IL-1R $a$ (e), IL-10 (f) and IL-13 (g), and the cytokines with dual roles IL-2 (h) and IL-6 (i) in each control or treatment group are graphed as the mean \pm standard error of the mean (SEM; $n=6$ for treatment groups; $n=3$ for naïve serum samples). $a^{*}$ or ${ }^{\wedge}$ denotes a $p$-value of $<0.05$ when compared to the vehicle control or naïve groups respectively.

nificantly reduced concentrations of IL-2 were measured in the SVF adipocyte co-culture secretion treatment group when compared to the vehicle control group (Figure 2(h)). The chemokine eotaxin was measured in significantly increased concentrations in the mice treated with secretions from the SVF co-cultured with adipocytes and the SVF alone when compared to both the vehicle control and naïve groups (Figure 3(a)). KC, MIP$1 \beta$ and GM-CSF were measured in significantly ncreased levels in the mice treated with SVF secretions versus the naïve mice (Figures 3(b), (e) and (f) respectively). MCP-1 and PDGF-bb levels were significantly increased in the serum of the mice treated with SVF adipocyte co-culture secretions when compared to the naïve mouse group (Figures 3(c) and (g) respectively). MIG was present in significantly reduced concentrations in the SVF adipocyte co-culture treatment group when compared to the vehicle control group (Figure 3(d)). VEGF was measured in significantly increased levels in the vehicle control group when compared to the naïve mice group (Figure 3(h)). Treatment with SVF adipocyte co-culture secretions significantly reduced the serum levels of VEGF of the mice when compared to the vehicle control (Figure 3(h)).

\section{DISCUSSION}

\subsection{SVF Adipocyte Co-Culture Secretions: A Promising Therapeutic for Inflammatory Conditions}

Rheumatoid arthritis is an autoimmune disorder characterized by chronic inflammation involving a shift towards the Th1 inflammatory response. The components of the Th1 response are involved in initiating and maintaining inflammation, including the production of the pro-inflammatory cytokines, IFN- $\gamma$, IL- $1 \alpha$, IL-1 $\beta$, IL-2, 
(a)

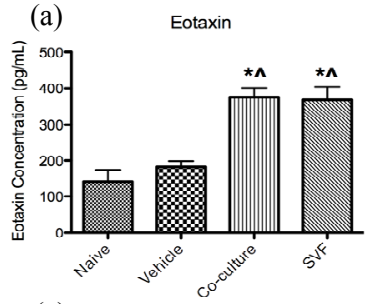

(c)

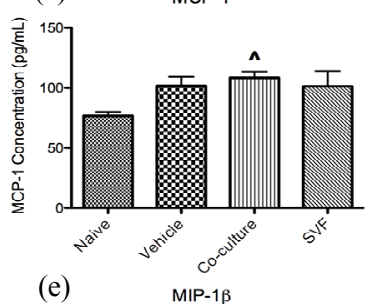

(e)

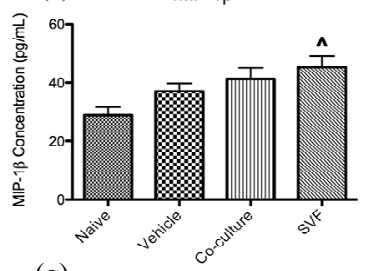

(g)

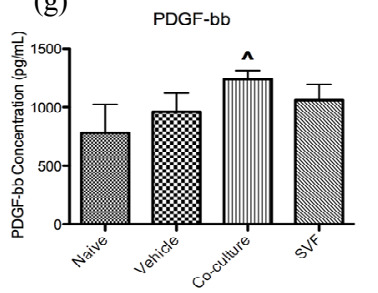

(b) KC-like

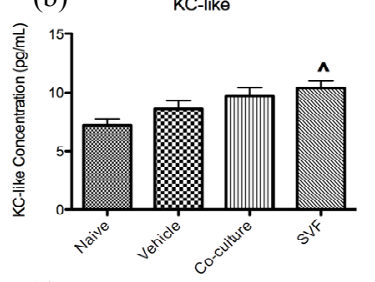

(d)

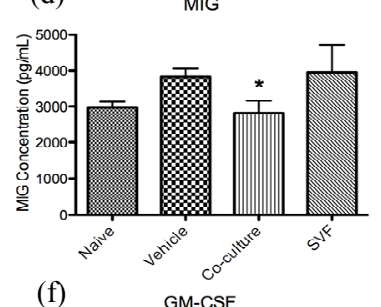

(f)

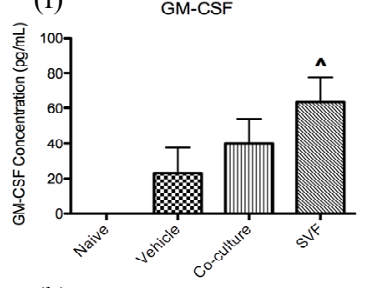

(h)

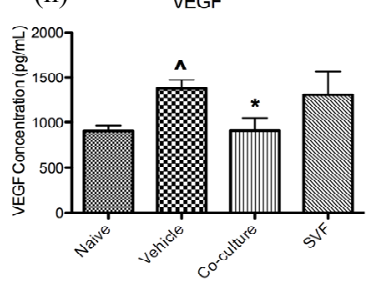

Figure 3. Serum levels of chemokines and growth factors measured in mice suffering from collagen antibody-induced arthritis following IV treatment with secretions from the SVF co-cultured with adipocytes, SVF secretions or a vehicle control. Serum samples were collected following euthanasia of the mice in all IV treatment groups at the completion of the trial and from naïve mice. The results of the chemokines, Eotaxin (a), KC (b), MCP-1 (c), MIG (d), and MIP-1 $\beta$ (e), and the growth factors GM-CSF (f), PDGF-bb (g) and VEGF (h), in each control or treatment group are graphed as the mean \pm standard error of the mean (SEM; $n=6$ for treatment groups; $n$ $=3$ for naïve serum samples). $\mathrm{a}^{*}$ or ${ }^{\wedge}$ denotes a $p$-value of $<0.05$ (students t-test) when compared to the vehicle control or naïve groups respectively.

IL-12 and TNF- $\alpha[25,26]$. These cytokines have been implicated in the pathogenesis of rheumatoid arthritis and are considered to contribute to cartilage degradation and bone erosion in this disease [27-29]. Routinely used glucocorticoid therapy promotes a shift towards the Th2 response [30], thereby counterbalancing the Th1 skewed environment seen in rheumatoid arthritis patients [31].

MSC therapy has been shown to have a positive effect on autoimmune conditions, primarily via secreted factors and their ability to induce peripheral tolerance $[32,33]$. We have previously demonstrated that the in vitro coculture of SVF with adipocytes results in the production

of a distinct secretion profile involving significantly increased concentrations of IL-1R $a$, IL-6, IL-7, IL-12, IFN- $\gamma$, IP-10, bFGF, G-CSF and VEGF when compared to culturing the SVF alone [8]. The in vitro effect of co-culture was carried over into the animal model used in this study and the IV administration of SVF adipocyte co-culture secretions achieved a superior therapeutic outcome. In this study the secretions administered were a complex mixture of hundreds of proteins and other signaling molecules, so the precise mechanism of action of this treatment is not known. A reduction of the clinical score to baseline at day 12 was observed in the CAIA mice treated with the SVF adipocyte co-culture treatment group. If it is assumed the circulating half-life of the human cytokines administered to the CAIA mice was minutes to hours [24], this reduction of the clinical score occurred 6 days after the human cytokines were cleared from the circulation. One of the mechanisms of action of MSCs is their ability to induce peripheral T cell tolerance by suppressing $\mathrm{T}$ cell proliferation and inducing $\mathrm{T}$ regulatory cell production [32,34]. The ability to induce $\mathrm{T}$ cell tolerance is an important therapeutic strategy for the effective treatment of autoimmune disorders. As secretions from MSCs are the major driver of the angiogenic, anti-apoptotic and anti-scarring properties of these cells, it is likely that MSC-derived cytokines also play some role in their ability to induce $\mathrm{T}$ cell tolerance. There is evidence that MSCs secrete cytokines in an environment or injury specific manner, with TGF- $\beta$ and IL-10 being important for inducing tolerance in autoimmune conditions such as RA [35,36]. As discussed by Ichim et al., (2010) the SVF contains a variety of cell types including $\mathrm{T}$ regulatory cells and high levels of MSCs [34]. Therefore, in this study, the SVF adipocyte co-culture and SVF secretion mixtures are likely to contain cytokines produced from both MSCs and T regulatory cells that are involved in inducing $\mathrm{T}$ cell tolerance. However, the therapeutics used in this study were complex mixtures of cytokines, which cannot respond to the local environment in the same dynamic way as implanted cells. It is possible, therefore, that the balance of cytokines administered induced only partial tolerance in these mice and that repeated doses would be required if symptoms recurred. This may explain the improvement in the therapeutic effect observed 4 days after the administration of the SVF adipocyte co-culture secretions. If SVF cell therapy was used as the therapeutic it is anticipated that the MSCs would induce $\mathrm{T}$ cell tolerance over a longer post-treatment period [34,37] Although it is likely that the effects observed with conditioned media will be similar to SVF cell therapy, there will be some differences. After the infusion of a mixed population of cells, such as SVF with adipocytes the subsequent therapeutic effect appears to be separated into two related phases. Firstly, the mixed population of cells secretes im- 
muno-modulatory cytokines, which have important therapeutic roles in reducing inflammation and pain. Secondly, the high levels of MSCs in the SVF are likely to embed in the host tissue, and control the microenvironment through their paracrine activities. It is anticipated that the therapeutic effect will be similar, but longer lasting, when human adipose-derived cell populations, such as SVF and adipocytes are used.

As observed in Figure 1(h) the clinical scores are increasing at the last time-point, which may indicate a recurrence of symptoms in some mice. In addition to the direct effect of cytokines on peripheral tolerance, it is also possible that the significantly higher levels of GCSF in the SVF adipocyte co-culture secretions over the SVF secretions administered to the CAIA mice may play some role. G-CSF has classically been used to stimulate mobilization of hematopoietic stem cells from the bone marrow into the circulation. More recently, mobilization of MSCs from the bone marrow has been demonstrated following administration of G-CSF [38]. Therefore, it is possible that following administration of the therapeutics used in this study, the high levels of G-CSF stimulated mobilization of some MSCs from the bone marrow, which may have contributed to the therapeutic outcome observed.

Our results suggest that the treatment with secretions from adipose-derived populations may be modulating the immune response and reducing the inflammation in these mice. At the serum level, it appears that the therapeutics used did not promote an exclusive Th1 or Th2 response. Instead, a significant increase in the pro-inflammatory cytokines IL- $1 \alpha$, IL- $1 \beta$ and TNF- $\alpha$ and a corresponding increase in the anti-inflammatory cytokines, IL-1R $a$, IL-10 $(\mathrm{p}<0.05)$ and IL-13 $(\mathrm{p}<0.05)$ were observed in the SVF adipocyte co-culture treatment group.

The role of IL-5 in rheumatoid arthritis is not well understood. However, whilst serum levels of IL-5 are generally $<10 \mathrm{pg} / \mathrm{mL}$, a significant increase is seen between early stage rheumatoid arthritis patients and healthy people [39]. Although the mouse serum levels of IL-5 in this study were higher than the levels seen in people, IL-5 was significantly increased in the vehicle control group whereas the mice treated with the therapeutics had approximately physiological levels. Rheumatoid arthritis patients have increased serum levels of the chemokine eotaxin [39], and higher levels been associated with less radiographic progression [40]. In this mouse model of rheumatoid arthritis, the mice in the vehicle control group that developed the most severe arthritis did not have elevated serum eotaxin levels above physiological levels. However, eotaxin levels were significantly increased in the mice that received the therapeutics in this study when compared to both the naïve and vehicle control mice (Figure 3(a)). Therefore whilst there are as- pects of the eotaxin and IL-5 results that are consistent with rheumatoid arthritis patients, the exact role of these two cytokines in CAIA is not well understood.

IL-2 and VEGF have been implicated in the pathogenesis of rheumatoid arthritis patients and are significantly increased in the serum of human patients with established rheumatoid arthritis [39]. Consistent with this observation, the serum levels of IL-2 and VEGF were significantly increased in the vehicle control group of mice that developed severe arthritis, when compared to physiological levels. IL-2 is a Th1 derived cytokine that contributes to disease progression by exacerbating Th1mediated disease states whilst recruiting other immune cells to the joint $[41,42]$. Increased expression of the IL-2 receptor on the surface of $\mathrm{T}$ cells is seen in a number of autoimmune disorders including rheumatoid arthritis [43]. It is known that blocking the ability of IL-2 to bind to its receptor does not affect natural immunity but can induce immunosuppression in a broad range of $\mathrm{T}$ cell mediated diseases [44]. Consequently, the IL-2 receptor is being targeted as a therapeutic strategy for the treatment of autoimmune disorders $[43,44]$. The administration of SVF adipocyte co-culture secretions produced an antiinflammatory effect and we observed significantly lower serum levels of IL-2 in this treatment group, consistent with the reduced severity of arthritis in these mice.

Although the etiology of rheumatoid arthritis disease development is not known, one of the characteristic features of early rheumatoid arthritis is an increase in the number of blood vessels in the pannus developing from the synovium [45]. The increased density of blood vessels is thought to result from a dis-regulation and imbalance between pro- and anti-angiogenic stimuli [45]. To this end, significantly increased circulating levels of VEGF are seen in patients with established rheumatoid arthritis when compared to healthy controls [39]. Consequently, blocking angiogenesis and the pathogenic mechanisms that stimulate neovascularization is an attractive therapeutic strategy and one that is being trialed. In fact, two studies have demonstrated that administration of the VEGF soluble receptor to mice suffering from collagen-induced arthritis reduces the severity of the disease $[46,47]$. In this study, the SVF adipocyte co-culture secretions contained significantly higher concentrations of VEGF than the levels present in the SVF secretions. However, we observed the largest improvement over the course of the study in the group of mice that received the SVF adipocyte co-culture secretions and this was accompanied by a significant reduction in serum levels of VEGF when compared to the vehicle control mice. Clearly the negative consequences resulting from angiogenesis require more than short-term elevated VEGF levels. The half-life of VEGF is approximately 34 minutes [48] and angiogenesis typically requires many 
days or weeks [49].

\subsection{Secretions-Based Therapy: Therapeutic Importance}

The use of autologous cell therapy, particularly with minimally manipulated adipose cell populations is becoming increasingly common as a therapy option for a variety of diseases including inflammatory conditions. The secretion of immuno-modulatory and trophic factors are a key aspect of the in vivo effect of MSCs and mixed cell populations [15]. We have previously demonstrated in vitro that mixed populations from adipose tissue, particularly those with SVF and adipocytes, produce distinct secretion profiles with high levels of growth factors and anti-inflammatory cytokines. The in vivo assessment of adipose SVF adipocyte co-culture secretions as not only a proxy for human SVF cell therapy, but also a potential stand-alone therapeutic in the CAIA model, enabled us to confirm our in vitro data. Although the exact mechanism of the therapeutic effect of the administration of SVF adipocyte co-culture secretions to CAIA mice is not known, no adverse effects were seen throughout the trial.

\section{CONCLUSION}

The administration of secretions from adipose-derived cell populations as a proxy for human adipose SVF cell therapy, do have the ability to reduce the severity of inflammatory diseases, although the mixture of cytokines is likely to be important. MSC therapy has been shown to be safe [50] and under the medical exemption regulatory category, autologous cell therapies, such as freshly isolated adipose-derived SVF with adipocytes, can be performed in-clinic under the supervision of a registered medical practitioner [1]. It is anticipated that the therapeutic effect will be similar, but longer lasting, when human adipose-derived cell populations, such as SVF and adipocytes are used. Additionally, the administration of these secretions to CAIA mice demonstrated a beneficial therapeutic effect thereby also providing a basis for future off-the-shelf secretion based products. There are a number of advantages to using off-the-shelf secretion based products as a therapeutic which includes the ease of transporting and storing these products and the ease of delivery via either the IV, IM, or sub-cutaneous route. However, due to the relatively short half-life of cytokines, it is likely that multiple treatments with secretion-based products will be required to sustain the therapeutic effect.

\section{REFERENCES}

[1] Mahalatchimy, A., Rial-Sebbag, E., Tournay, V., et al. (2012) The legal landscape for advanced therapies: Material and institutional implementation of European Union rules in France and the United Kingdom. Journal of Law and Society, 39, 131-149.

doi:10.1111/j.1467-6478.2012.00574.x

[2] Nakagami, H., Maeda, K., Morishita, R., et al. (2005) Novel autologous cell therapy in ischemic limb disease through growth factor secretion by cultured adipose tissue-derived stromal cells. Arteriosclerosis, Thrombosis, and Vascular Biology, 25, 2542-2547. doi:10.1161/01.ATV.0000190701.92007.6d

[3] Chopp, M. and Li, Y. (2002) Treatment of neural injury with marrow stromal cells. Lancet Neurology, 1, 92-100. doi:10.1016/S1474-4422(02)00040-6

[4] Rehman, J., Traktuev, D., Li, J., et al. (2004) Secretion of angiogenic and antiapoptotic factors by human adipose stromal cells. Circulation, 109, 1292-1298. doi:10.1161/01.CIR.0000121425.42966.F1

[5] Nishimura, S., Manabe, I., Nagasaki, M., et al. (2009) $\mathrm{CD} 8+$ effector $\mathrm{T}$ cells contribute to macrophage recruitment and adipose tissue inflammation in obesity. Nature Medicine, 15, 914-920. doi:10.1038/nm.1964

[6] Poggi, M., Jager, J., Paulmyer-Lacroix, O., et al. (2009) The inflammatory receptor CD40 is expressed on human adipocytes: Contribution to crosstalk between lymphocytes and adipocytes. Diabetologia, 52, 1152-1163. doi:10.1007/s00125-009-1267-1

[7] Suganami, T., Nishida, J. and Ogawa, Y. (2005) A paracrine loop between adipocytes and macrophages aggravates inflammatory changes - Role of free fatty acids and tumor necrosis factor alpha. Arteriosclerosis, Thrombosis, and Vascular Biology, 25, 2062-2068. doi:10.1161/01.ATV.0000183883.72263.13

[8] Blaber, S.P., Webster, R.A., Hill, C.J., et al. (2012) Analysis of in vitro secretion profiles from adiposederived cell populations. Journal of Translational Medicine, 10, 172-188. doi:10.1186/1479-5876-10-172

[9] Leonard, J.P., Sherman, M.L., Fisher, G.L., et al. (1997) Effects of single-dose interleukin-12 exposure on interleukin-12-associated toxicity and interferon-gamma production. Blood, 90, 2541-2548.

[10] Vial, T. and Descotes, J. (1995) Immune-mediated sideeffects of cytokines in humans. Toxicology, 105, 31-57. doi:10.1016/0300-483X(95)03124-X

[11] Tayal, V. and Kalra, B.S. (2008) Cytokines and anti-cytokines as therapeutics-An update. European Journal of Pharmacology, 579, 1-12.

[12] Gabay, C., Lamacchia, C. and Palmer, G. (2010) IL-1 pathways in inflammation and human diseases. Nature Reviews Rheumatology, 6, 232-241. doi:10.1038/nrrheum.2010.4

[13] Asahara, T., Bauters, C., Zheng, L.P., et al. (1995) Synergistic effect of vascular endothelial growth factor and basic fibroblast growth factor on angiogenesis in vivo. Circulation, 92, 365-371. doi:10.1161/01.CIR.92.9.365

[14] Cao, R., Brakenhielm, E., Pawliuk, R., et al. (2003) Angiogenic synergism, vascular stability and improvement of hind-limb ischemia by a combination of PDGF-BB and FGF-2. Nature Medicine, 9, 604-613. doi: $10.1038 / \mathrm{nm} 848$

[15] Meirelles Lda, S., Fontes, A.M., Covas, D.T., et al. (2009) 
Mechanisms involved in the therapeutic properties of mesenchymal stem cells. Cytokine \& Growth Factor Reviews, 20, 419-427. doi:10.1016/j.cytogfr.2009.10.002

[16] Oh, J.Y., Kim, M.K., Shin, M.S., et al. (2008) The antiinflammatory and anti-angiogenic role of mesenchymal stem cells in corneal wound healing following chemical injury. Stem Cells, 26, 1047-1055. doi:10.1634/stemcells.2007-0737

[17] Timmers, L., Lim, S.K., Hoefer, I.E., et al. (2011) Human mesenchymal stem cell-conditioned medium improves cardiac function following myocardial infarction. Stem Cell Research, 6, 206-214. doi:10.1016/j.scr.2011.01.001

[18] Cho, Y.J., Song, H.S., Bhang, S., et al. (2012) Therapeutic effects of human adipose stem cell-conditioned medium on stroke. Journal of Neuroscience Research, 90, 1794-17802. doi:10.1002/jnr.23063

[19] Williams, R.O., Inglis, J.J., Simelyte, E., et al. (2005) Analysing the effect of novel therapies on cytokine expression in experimental arthritis. International Journal of Experimental Pathology, 86, 267-278. doi:10.1111/j.0959-9673.2005.00443.x

[20] Nandakumar, K.S. and Holmdahl, R. (2006) Antibodyinduced arthritis: Disease mechanisms and genes involved at the effector phase of arthritis. Arthritis Research \& Therapy, 8, 223. doi:10.1186/ar2089

[21] Terato, K., Harper, D.S., Griffiths, M.M., et al. (1995) Collagen-induced arthritis in mice: Synergistic effect of $E$. coli lipopolysaccharide bypasses epitope specificity in the induction of arthritis with monoclonal antibodies to type II collagen. Autoimmunity, 22, 137-147. doi:10.3109/08916939508995311

[22] Morris, C.J. (2003) Carrageenan-induced paw edema in the rat and mouse. Methods in Molecular Biology, 225, 115-121.

[23] Khachigian, L.M. (2006) Collagen antibody-induced arthritis. Nature Protocols, 1, 2512-2516. doi:10.1038/nprot.2006.393

[24] Schaller, J., Gerber, S., Kaempfer, U., et al. (2008) Human blood plasma proteins: Structure and function. John Wiley \& Sons, West Sussex. doi:10.1002/9780470724378

[25] Verhoef, C.M., van Roon, J.A., Vianen, M.E., et al. (1999) The immune suppressive effect of dexamethasone in rheumatoid arthritis is accompanied by upregulation of interleukin 10 and by differential changes in interferon gamma and interleukin 4 production. Annals of the Rheumatic Diseases, 58, 49-54. doi:10.1136/ard.58.1.49

[26] Mosmann, T.R. and Sad, S. (1996) The expanding universe of T-cell subsets: Th1, Th2 and more. Immunology Today, 17, 138-146. doi:10.1016/0167-5699(96)80606-2

[27] Wilbrink, B., Holewijn, M., Bijlsma, J.W., et al. (1993) Suppression of human cartilage proteoglycan synthesis by rheumatoid synovial fluid mononuclear cells activated with mycobacterial 60-kd heat-shock protein. Arthritis \& Rheumatism, 36, 514-518. doi:10.1016/0167-5699(96)80606-2

[28] Schulze-Koops, H., Lipsky, P.E., Kavanaugh, A.F., et al. (1995) Elevated Th1- or Th0-like cytokine mRNA in peripheral circulation of patients with rheumatoid arthritis.
Modulation by treatment with anti-ICAM-1 correlates with clinical benefit. The Journal of Immunology, 155, 50295037.

[29] Dolhain, R.J.E.M., vander Heiden, A.N., terHaar, N.T., et al. (1996) Shift toward T lymphocytes with a T helper 1 cytokine-secretion profile in the joints of patients with rheumatoid arthritis. Arthritis \& Rheumatism, 39, 19611969. doi:10.1002/art.1780391204

[30] Agarwal, S.K. and Marshall, G.D. (2001) Dexamethasone promotes type 2 cytokine production primarily through inhibition of type 1 cytokines. Journal of Interferon \& Cytokine Research, 21, 147-155. doi:10.1089/107999001750133159

[31] Miossec, P. and van den Berg, W. (1997) Th1/Th2 cytokine balance in arthritis. Arthritis \& Rheumatism, 40, 2105-2115. doi:10.1002/art.1780401203

[32] Zappia, E., Casazza, S., Pedemonte, E., et al. (2005) Mesenchymal stem cells ameliorate experimental autoimmune encephalomyelitis inducing T-cell anergy. Blood, 106, 1755-1761. doi:10.1182/blood-2005-04-1496

[33] Gerdoni, E., Gallo, B., Casazza, S., et al. (2007) Mesenchymal stem cells effectively modulate pathogenic immune response in experimental autoimmune encephalomyelitis. Annals of Neurology, 61, 219-227. doi:10.1002/ana.21076

[34] Ichim, T.E., Harman, R.J., Min, W.P., et al. (2010) Autologous stromal vascular fraction cells: A tool for facilitating tolerance in rheumatic disease. Cellular Immunology, 264, 7-17. doi:10.1016/j.cellimm.2010.04.002

[35] Nasef, A., Chapel, A., Mazurier, C., et al. (2007) Identification of IL-10 and TGF-beta transcripts involved in the inhibition of T-lymphocyte proliferation during cell contact with human mesenchymal stem cells. Gene Expression, 13, 217-226. doi:10.3727/000000006780666957

[36] Li, H., Guo, Z., Jiang, X., et al. (2008) Mesenchymal stem cells alter migratory property of $\mathrm{T}$ and dendritic cells to delay the development of murine lethal acute graft-versus-host disease. Stem Cells, 26, 2531-2541. doi:10.1634/stemcells.2008-0146

[37] Mokarizadeh, A., Delirezh, N., Morshedi, A., et al. (2012) Microvesicles derived from mesenchymal stem cells: Potent organelles for induction of tolerogenic signaling. Immunology Letters, 147, 47-54. doi:10.1016/j.imlet.2012.06.001

[38] Deng, J., Zou, Z.M., Zhou, T.L., et al. (2011) Bone marrow mesenchymal stem cells can be mobilized into peripheral blood by G-CSF in vivo and integrate into traumatically injured cerebral tissue. Neurological Sciences, 32, 641-651. doi:10.1007/s10072-011-0608-2

[39] Kokkonen, H., Soderstrom, I., Rocklov, J., et al. (2010) Up-regulation of cytokines and chemokines predates the onset of rheumatoid arthritis. Arthritis \& Rheumatism, 62 , 383-391.

[40] Syversen, S.W., Goll, G.L., Haavardsholm, E.A., et al. (2008) A high serum level of eotaxin (CCL 11) is associated with less radiographic progression in early rheumatoid arthritis patients. Arthritis Research \& Therapy, 10, R28. doi:10.1186/ar2381 
[41] Feldmann, M. and Maini, R.N. (1999) The role of cytokines in the pathogenesis of rheumatoid arthritis. Rheumatology, 38, 3-7.

[42] Thornton, S., Boivin, G.P., Kim, K.N., et al. (2000) Heterogeneous effects of IL-2 on collagen-induced arthritis. Journal of Immunology, 165, 1557-1563.

[43] Morris, J.C. and Waldmann, T.A. (2000) Advances in interleukin 2 receptor targeted treatment. Annals of the Rheumatic Diseases, 59, 109-114. doi:10.1136/ard.59.suppl 1.i109

[44] Brok, H.P.M., Tekoppele, J.M., Hakimi, J., et al. (2001) Prophylactic and therapeutic effects of a humanized monoclonal antibody against the IL-2 receptor (DACLIZUMAB) on collagen-induced arthritis (CIA) in rhesus monkeys. Clinical \& Experimental Immunology, 124, 134-141. doi:10.1046/j.1365-2249.2001.01487.x

[45] Paleolog, E.M. and Fava, R.A. (1998) Angiogenesis in rheumatoid arthritis: Implications for future therapeutic strategies. Springer Seminars in Immunopathology, 20, 73-94. doi:10.1007/BF00832000

[46] Afuwape, A.O., Feldmann, M. and Paleolog, E.M. (2003) Adenoviral delivery of soluble VEGF receptor 1 (sFlt-1) abrogates disease activity in murine collagen-induced arthritis. Gene Therapy, 10, 1950-1960.

\section{doi:10.1038/sj.gt.3302104}

[47] Miotla, J., Maciewicz, R., Kendrew, J., et al. (2000) Treatment with soluble VEGF receptor reduces disease severity in murine collagen-induced arthritis. Laboratory Investigation, 80, 1195-1205. doi:10.1038/labinvest.3780127

[48] Eppler, S.M., Combs, D.L., Henry, T.D., et al. (2002) A target-mediated model to describe the pharmacokinetics and hemodynamic effects of recombinant human vascular endothelial growth factor in humans. Clinical Pharmacology \& Therapeutics, 72, 20-32. doi:10.1067/mcp.2002.126179

[49] Pettersson, A., Nagy, J.A., Brown, L.F., et al. (2000) Heterogeneity of the angiogenic response induced in different normal adult tissues by vascular permeability factor/vascular endothelial growth factor. Laboratory Investigation, 80, 99-115. doi:10.1038/labinvest.3780013

[50] Lalu, M.M., McIntyre, L., Pugliese, C., et al. (2012) Safety of cell therapy with mesenchymal stromal cells (SafeCell): A systematic review and meta-analysis of clinical trials. PLoS One, 7, e47559. doi:10.1371/journal.pone.0047559 